\title{
On the optimum antenna pattern for widebeam radar reflectivity estimation
}

\author{
F. Gerbl and E. M. Biebl
}

Fachgebiet Höchstfrequenztechnik, Technische Universität München, Munich, Germany

\begin{abstract}
Noise is a limiting factor in radar systems. The power received from a target depends on the target reflectivity varying with the aspect angle, the target range and the antenna pattern, amongst others. Inevitably, an estimation algorithm for the mean target reflectivity weights noise contributions from greater aspect angles stronger than such from smaller aspect angles due to the target range increasing with increasing aspect angle. However, as an opposed effect, due to the supposed equidistant rather than equiangular sampling along the linear aperture, noise contributions for greater aspect angles have lower influence than those for smaller aspect angles. In the paper, the signal-to-noise ratio for a linear, equidistantly sampled aperture and an arbitrary antenna pattern is determined. Moreover, an upper limit for the achievable signal-to-noise ratio is given, and the antenna pattern maximizing the signal-to-noise ratio is derived.
\end{abstract}

\section{Introduction}

Noise is a limiting factor in radar systems. The power received from a target depends on the target reflectivity varying with the aspect angle, the target range and the antenna pattern, amongst others. Directing power only into a narrow region of space, i.e. using a high gain antenna, yields higher received power and higher azimuth resolution than transmitting power to a broader region of space. However, using a narrow antenna beam to linearly scan a target scene limits the aspect angles, from which targets with generally unknown orientation are illuminated. Widening the antenna beam and processing the received signal in an appropriate manner, an image containing estimates of the mean target reflectivities over the aspect angle can be obtained. For wide antenna beams, the range of a target as measured from the

Correspondence to: F. Gerbl

(gerbl@tum.de) radar system, and therefore the power received from even an omnidirectionally scattering target, varies as the radar system passes by the target.

\section{Setup}

The radar system is moved along a straight path, passing the scene consisting of targets to be imaged. The orientation of the antenna remains unchanged throughout the passing. The system transmits a signal which is reflected by the objects in the scene and in turn received by the system. The amplitude and phase of the received signal are determined and sampled equidistantly along the straight path. The radar system is assumed to be monostatic. Figure 1 illustrates the setup and indicates the changes the signal undergoes during the passing. Distance and aspect angle and therefore level of free space attenuation, phase shift and the influence of the antenna pattern vary as the antenna passes by the target.

\section{Coordinate system}

The basis for subsequent considerations are coordinate systems as depicted in Fig. 2. The coordinate systems are antenna-centered. The trajectory of the target in the antennacentered coordinate systems is a straight line in case the antenna is moved in a certain distance to the target on a straight line. It is assumed that the relative motion of the target occurs with respect to the $x$ coordinate, i.e. $y$ and $z$ remain constant. Since antenna patterns are usually given in spherical coordinates, a spherical coordinate system is attached to the Cartesian one. The spherical coordinate system is oriented in such a way that its meridians intersect on the $x$ axis. This orientation allows to describe the position of the target by only one varying angle coordinate, $\alpha$, while the other an-

Published by Copernicus Publications on behalf of the URSI Landesausschuss in der Bundesrepublik Deutschland e.V. 


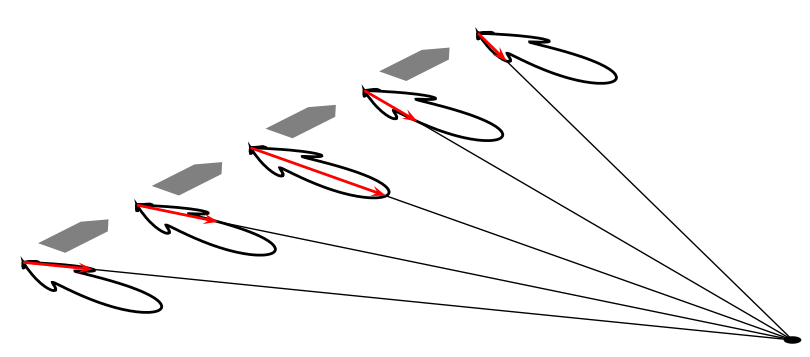

Fig. 1. Sketch of the setup.

gle coordinate, $\beta$, remains constant at the value

$\beta_{\mathrm{t}}=\arctan \frac{z_{\mathrm{t}}}{y_{\mathrm{t}}}$

where $y_{\mathrm{t}}$ and $z_{\mathrm{t}}$ are the $y$ and $z$ coordinates of the target. In Fig. 2, a possible trajectory of a target is indicated with a red line parallel to the $x$ axis.

\section{Signal processing}

The received signal is a function of the aspect angle $\alpha$ and time $t$ and can be written as

$$
\begin{aligned}
s_{\mathrm{RX}}(\alpha, t)= & a \cdot \rho(\alpha) \cdot \frac{1}{r^{2}(\alpha)} \cdot D_{\mathrm{A}}^{2}(\alpha) \cdot \exp \{-\mathrm{j} 2 k r(\alpha)\} \\
& +n_{\Re}(t)+\mathrm{j} n_{\Im}(t)
\end{aligned}
$$

in the presence of noise, assuming there is only one single point-like target. $a$ is a constant determined by system parameters like radiated power, mixer losses, and others. $\rho$ is the reflectivity of the target, $r$ the distance between antenna and target, $D_{\mathrm{A}}$ the antenna amplitude pattern, $k$ the wave number, and $n_{\mathfrak{R}}(t)$ and $n_{\Im}(t)$ are the noise contributions in real and imaginary part of the received signal, respectively. Actually, the antenna pattern is a function of both $\alpha$ and $\beta$. However, since the antenna pattern is assumed to be constant over $\beta$ except for a rectangular window that will be accounted for by a proper choice of integration limits, the pattern will be denoted as a function of only $\alpha$ instead of $\alpha$ and $\beta$ in the majority of cases in order to avoid unnecessarily long expressions.

Compensating the aforementioned effects and integrating (in reality summarizing) the compensated signals yields a noisy estimate $\hat{\bar{\rho}}_{\alpha_{0}}$ of the mean reflectivity over the angle $\alpha$ within $\pm \alpha_{0}$

$a \cdot 2 \alpha_{0} \cdot \hat{\bar{\rho}}_{\alpha_{0}}=\int_{-\alpha_{0}}^{\alpha_{0}} a \cdot \rho(\alpha) d \alpha+\int_{-\alpha_{0}}^{\alpha_{0}} n^{\prime}(\alpha, t) d \alpha$

with

$n^{\prime}(\alpha, t)=\left(n_{\Re}(t)+\mathrm{j} n_{\Im}(t)\right) \cdot r^{2}(\alpha) \cdot \frac{1}{D_{\mathrm{A}}^{2}(\alpha)} \cdot \exp \{\mathrm{j} 2 k r(\alpha)\}$,

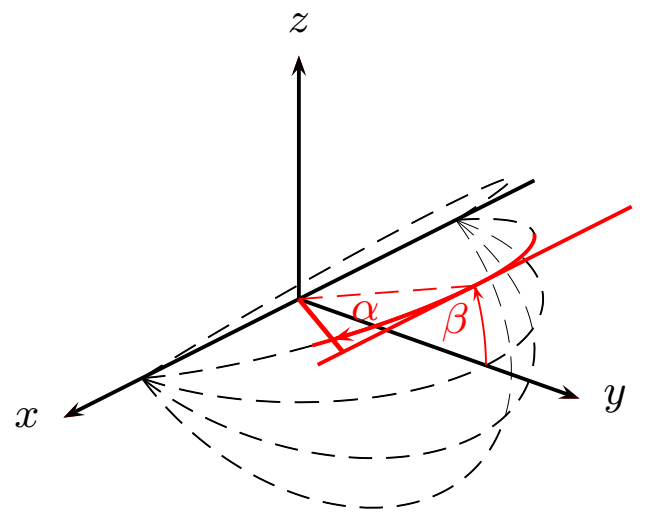

Fig. 2. Antenna-centered coordinate systems and trajectory of a target.

where the estimate consists of the desired signal and a noise contribution. It is desirable to minimize the noise contribution in order to maximize the signal-to-noise ratio.

Throughout the paper, integrals are used for the discussion where possible rather than sums. There remains an uncertainty in the estimation of the mean reflectivity due to the non-zero distance between two samples. However, the spacing has to be chosen relatively small in order to avoid aliasing in case of wide antenna beams. It is assumed that the spacing is so small that sum and integral can be regarded as equal.

For that pixel of the reflectivity map that corresponds to the true target location, the signal-to-noise ratio of the processing result is

$\mathrm{SNR}=\frac{S}{N}$

where

$S=\left|\int_{-\alpha_{0}}^{\alpha_{0}} a \cdot \rho(\alpha) d \alpha\right|^{2}=\left|a \cdot 2 \alpha_{0} \cdot \bar{\rho}_{\alpha_{0}}\right|^{2}$

is the power of the desired signal and

$N=\mathrm{E}\left\{\left|\int_{-\alpha_{0}}^{\alpha_{0}} n^{\prime}(\alpha, t) d \alpha\right|^{2}\right\}$

is the mean power of the noise contribution after processing. $\mathrm{E}\{\cdot\}$ denotes the expected value. Note that $N$ is not the power of the noise contributions $n_{\Re}(t)$ and $n_{\Im}(t)$ but that of the noise in the processing result. It is shown in Appendix A that the SNR can be written as

$$
\mathrm{SNR}=\frac{4 \alpha_{0}^{2}\left|a \cdot \bar{\rho}_{\alpha_{0}}\right|^{2}}{\Delta x \cdot r_{0}^{3} \cdot P_{\mathrm{n}}^{\prime} \cdot \int_{-\alpha_{0}}^{\alpha_{0}} \frac{1}{D^{2}(\alpha) \cos ^{2}(\alpha)} d \alpha},
$$


where $\bar{\rho}_{\alpha_{0}}$ is the true mean value of the target reflectivity within $\pm \alpha_{0}, \Delta x$ the spatial distance between two samplings, and $r_{0}$ the minimum distance of the target to the $x$-axis. The power of the noise term $n_{\mathfrak{R}}(t)$ is assumed to be equal to that of $n_{\Im}(t)$. The sum of those noise powers is proportional to $P_{\mathrm{n}}^{\prime} . D(\alpha)=D_{\mathrm{A}}^{2}(\alpha)$ is the antenna power pattern.

\section{Optimization}

From Eq. (8) it is obvious that the SNR is influenced by the antenna pattern. The antenna pattern is to be optimized in that sense that the SNR after the signal processing is maximized, i.e. the expression

$$
\int_{-\alpha_{0}}^{\alpha_{0}} \frac{1}{D^{2}(\alpha) \cos ^{2}(\alpha)} d \alpha
$$

in the denominator of Eq. (8) is to be minimized. The boundary condition for the optimization is generally

$$
\iint D(\Omega) d \Omega=4 \pi
$$

and particularly for the coordinate system used here

$$
\int_{-\pi}^{\pi} \int_{-\pi / 2}^{\pi / 2} D(\alpha, \beta) \cos (\alpha) d \alpha d \beta=4 \pi .
$$

i.e. the integral of the antenna pattern over all solid angle elements of a sphere has to be equal to $4 \pi$.

The first step to maximizing the SNR is to restrict the radiation to only those regions of space from where signals will be processed. For a given maximum processing angle $\alpha_{0}$, the antenna pattern has to vanish for $|\alpha|>\alpha_{0}$. For the following considerations it is assumed that the antenna pattern does not vary with the angle $\beta$ except for the fact that the pattern vanishes for $|\beta|>\beta_{0}$ resulting in a limited field of view, within which the SNR is independent of the target's location with respect to the related angle $\beta$. Under the prerequisites of a pattern vanishing for $|\alpha|>\alpha_{0}$ and $|\beta|>\beta_{0}$ and being constant with respect to $\beta$ inside its support region, the boundary condition can be written as

$$
2 \beta_{0} \int_{-\alpha_{0}}^{\alpha_{0}} D(\alpha) \cos (\alpha) d \alpha=4 \pi .
$$

It is shown in Appendix B that the optimum antenna pattern, $D_{\text {opt }, \alpha_{0}}$, for a given maximum processing angle $\alpha_{0}$ that maximizes the SNR is

$$
D_{\text {opt }, \alpha_{0}}(\alpha)= \begin{cases}\frac{\pi}{\alpha_{0} \beta_{0}} \cdot \frac{1}{\cos (\alpha)} & \text { for }|\alpha| \leq \alpha_{0} \text { and }|\beta| \leq \beta_{0} \\ 0 & \text { else }\end{cases}
$$

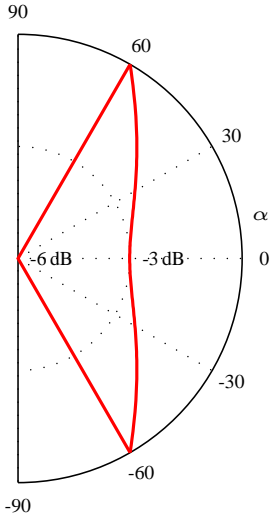

Fig. 3. Normalized optimum pattern $D_{\mathrm{opt}, \alpha_{0}}(\alpha)$ for $\alpha_{0}=60^{\circ}$.

For every maximum processing angle $\alpha_{0}$, a different antenna pattern is optimum. Using the respective pattern, the maximum achievable signal-to-noise ratio $\mathrm{SNR}_{\max }$ can be determined with Eqs. (8) and (13) to be

$$
\mathrm{SNR}_{\max }=\frac{2 \pi^{2}\left|a \bar{\rho}_{\alpha_{0}}\right|^{2}}{\Delta x \cdot r_{0}^{3} \cdot P_{\mathrm{n}}^{\prime} \cdot \alpha_{0} \cdot \beta_{0}^{2}} .
$$

The maximum achievable signal-to-noise ratio decreases with increasing antenna beam width. Nevertheless, there might be reasons (e.g. decreasing the probability of missing a target that reflects strongly anisotropically, increasing azimuth resolution) to use wide antenna beams. The signal-tonoise ratio is only one figure of merit amongst others. Using the appropriate optimum pattern yields the maximum achievable SNR for a given $\alpha_{0}$. Figure 3 shows exemplarily the optimum pattern for $\alpha_{0}=60^{\circ}$.

\section{Benefit of the findings}

Equation (8) allows the determination of the achievable SNR for a given antenna pattern and a certain maximum processing angle $\alpha_{0}$ that might be dictated by special needs of a certain application. Equation (14) provides a benchmark for the antenna under consideration. The Figs. 4, 5 and 6 show three different antenna patterns and the resulting normalized signal-to-noise ratios for different maximum processing angles $\alpha_{0}$ along with the normalized maximum achievable signal-to-noise ratios using the appropriate optimum patterns matched to the respective maximum processing angles. Reference for the normalization is the signal-to-noise ratio obtainable for $\alpha_{0}=90^{\circ}$ with the respective optimum pattern.

Figure 4 shows the normalized pattern (red) of an antenna that radiates power uniformly into all directions of the hemisphere. Nominally, this antenna has a beam width of $\pm 90^{\circ}$. However, its optimum maximum processing angle is $\alpha_{0} \approx 54^{\circ}$. The maximum signal-to-noise ratio achievable with 

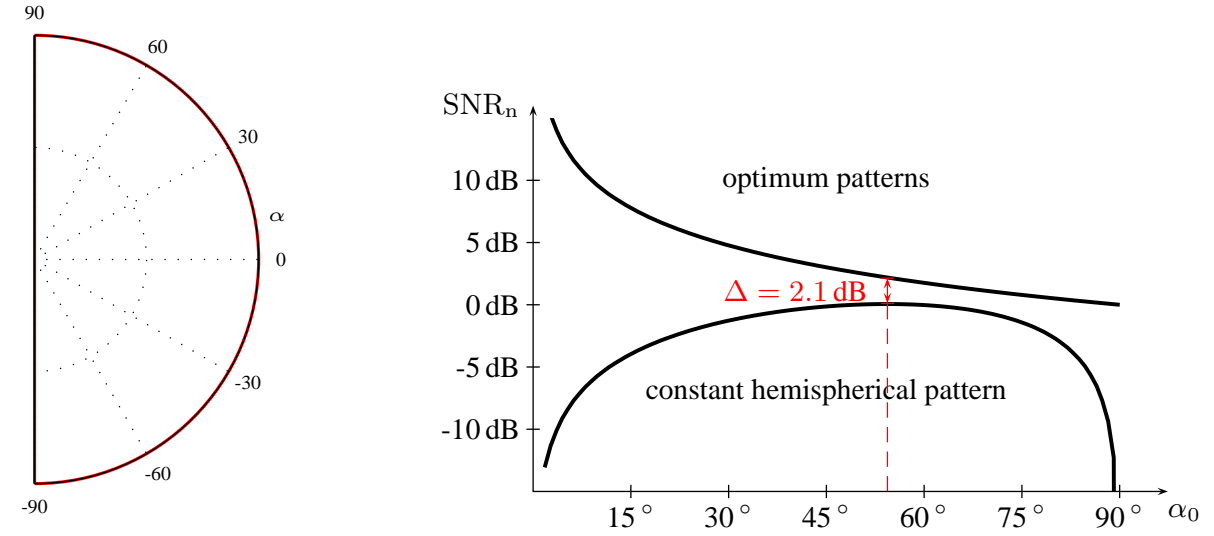

Fig. 4. Plot of a constant hemispherical pattern (red) and the resulting normalized signal-to-noise ratios compared to the optimum pattern.
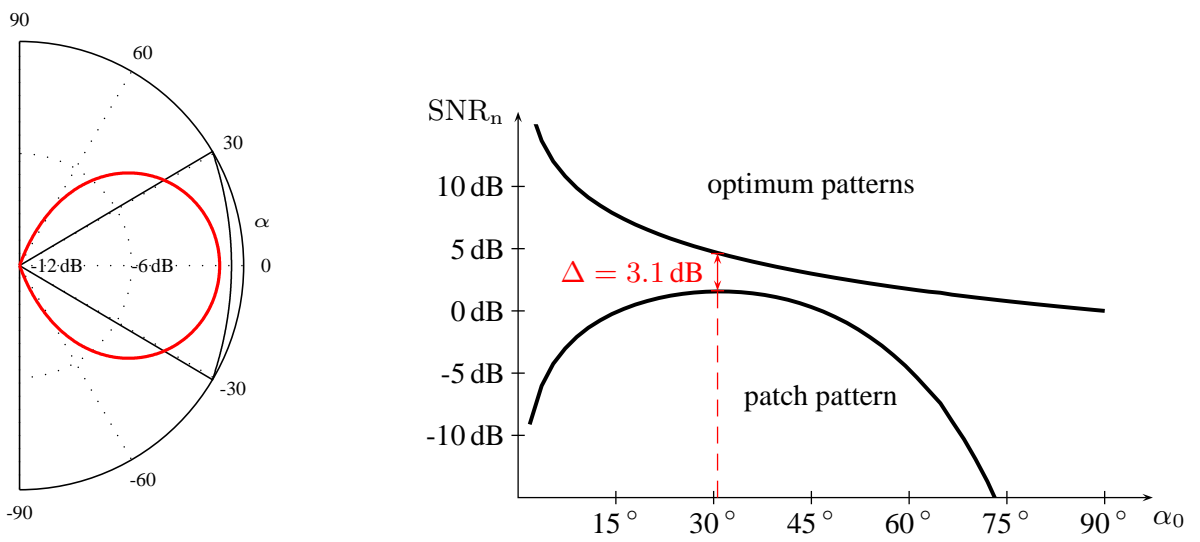

Fig. 5. Plot of the pattern of a patch antenna (red) and the resulting normalized signal-to-noise ratios.

this pattern is about $2.1 \mathrm{~dB}$ below that achievable with the respective optimum pattern.

Figure 5 shows the normalized pattern (red) of a patch antenna. Its optimum maximum processing angle is $\alpha_{0} \approx 31^{\circ}$. The pattern plot also shows the pattern (black) of the optimum pattern for $\alpha_{0}=31^{\circ}$. The maximum signal-to-noise ratio achievable with the patch pattern is about $3.1 \mathrm{~dB}$ below that achievable with the respective optimum pattern.

Figure 6 shows a fictitious pattern (red) as it might emerge during the design process of an antenna. Its optimum maximum processing angle is $\alpha_{0} \approx 31^{\circ}$ as for the patch antenna considered before. The pattern plot also shows the pattern (black) of the optimum pattern for $\alpha_{0}=31^{\circ}$. The maximum signal-to-noise ratio achievable with this fictitious pattern is about $1 \mathrm{~dB}$ below that achievable with the respective optimum pattern. The reason for the drop-off of the signal-tonoise ratio for angles above the optimum angle is that only a very small fraction of the energy is radiated into the respective directions and the compensation of signals received from those directions causes strong noise contributions due to a multiplication with the reciprocal of the almost vanishing antenna pattern.

The curve of the maximum achievable signal-to-noise ratio given by Eq. (14) can be used advantageously to judge the quality of the approximation of a desired antenna pattern by the current design.

\section{Conclusions}

In this paper, the signal-to-noise ratio of radar reflectivity estimation by sampling a linear aperture equidistantly and processing the received signals properly has been derived. It has been shown that the signal-to-noise ratio depends on the antenna pattern, amongst others. The optimum patterns, in that sense that they maximize the signal-to-noise ratio, as a function of the aspect angle and the maximum processing angle have been determined. The maximum achievable signal-tonoise ratio using the optimum patterns is stated. The findings 

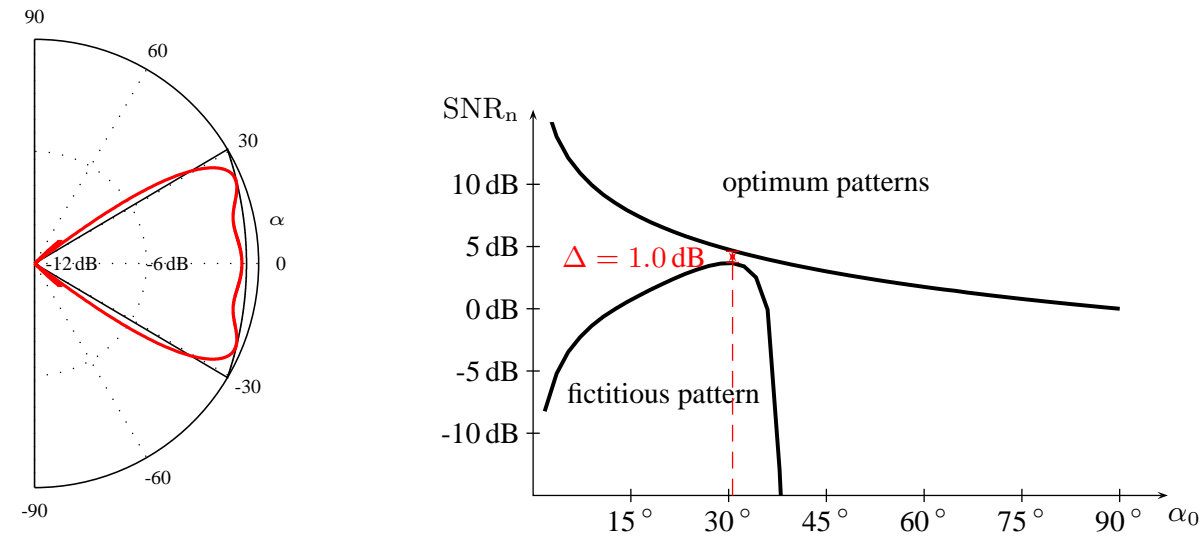

Fig. 6. Plot of a fictitious pattern (red) and the resulting normalized signal-to-noise ratios.

can be used to evaluate the performance of existing antennas for the task of estimating the radar reflectivity within different maximum processing angles. The results show that the signal-to-noise ratio achievable with a certain pattern as it might emerge in one of the iterations of an antenna design process does not depend on single values of the pattern but on an integral over a function of the antenna pattern. Thus, the difference of the signal-to-noise ratio achievable with the pattern under consideration and that achievable with the appropriate optimum pattern can be used as a figure of merit for the evaluation of an antenna pattern.

\section{Appendix A Determination of the signal-to-noise ratio}

Content of this section is the derivation of Eq. (8). Samples of the received signal are taken equidistantly at discrete positions along a straight line. Therefore, the number of samples per angle element is greater for greater angles $\alpha$ than for smaller ones. To account for this fact, the statistical evaluation of the noise contribution has to be done after the substitution of $\alpha$ by $x$. With

$\alpha=\arctan \frac{x}{r_{0}}, \quad \frac{d \alpha}{d x}=\frac{r_{0}}{r_{0}^{2}+x^{2}}$

Equation (7) can be written as

$N=\mathrm{E}\left\{\left|\int_{-x_{0}}^{x_{0}} n^{\prime}(\alpha(x), t) \frac{r_{0}}{r_{0}^{2}+x^{2}} d x\right|^{2}\right\}$.

Using

$r_{0}^{2}+x^{2} \equiv r^{2}(\alpha(x))$

and

$n^{\prime \prime}(\alpha(x), t)=\frac{n^{\prime}(\alpha(x), t)}{r^{2}(\alpha(x))}$, the noise power after processing reads as

$$
N=r_{0}^{2} \mathrm{E}\left\{\left|\int_{-x_{0}}^{x_{0}} n^{\prime \prime}(\alpha(x), t) d x\right|^{2}\right\}
$$

and consequently

$$
\begin{aligned}
N=r_{0}^{2} \mathrm{E}\left\{\left(\int_{-x_{0}}^{x_{0}} \Re\left\{n^{\prime \prime}(\alpha(x), t)\right\} d x\right)^{2}\right. \\
\left.+\left(\int_{-x_{0}}^{x_{0}} \Im\left\{n^{\prime \prime}(\alpha(x), t)\right\} d x\right)^{2}\right\} \\
=r_{0}^{2}\left(\mathrm{E}\left\{X_{1}^{2}\right\}+\mathrm{E}\left\{X_{2}^{2}\right\}\right)
\end{aligned}
$$

with

$X_{1}=\int_{-x_{0}}^{x_{0}} \Re\left\{n^{\prime \prime}(\alpha(x), t)\right\} d x$

and

$X_{2}=\int_{-x_{0}}^{x_{0}} \mathfrak{s}\left\{n^{\prime \prime}(\alpha(x), t)\right\} d x$.

The integrands are

$$
\begin{aligned}
& \Re\left\{n^{\prime \prime}(\alpha(x), t)\right\}= \\
& \frac{n_{\Re}(t) \cos (2 k r(\alpha(x)))-n_{\Im}(t) \sin (2 k r(\alpha(x)))}{D(\alpha(x))}
\end{aligned}
$$

and

$$
\begin{aligned}
& \Im\left\{n^{\prime \prime}(\alpha(x), t)\right\}= \\
& \frac{n_{\Re}(t) \sin (2 k r(\alpha(x)))+n_{\Im}(t) \cos (2 k r(\alpha(x)))}{D(\alpha(x))} .
\end{aligned}
$$


$n_{\Re}(t)$ and $n_{\Im}(t)$ are zero-mean. Therefore, the sum of several samples of those noise processes is zero-mean:

$\mathrm{E}\left\{X_{1}\right\}=\mathrm{E}\left\{X_{2}\right\}=0$.

Since generally

$$
\mathrm{E}\left\{X^{2}\right\}=\operatorname{Var}\{X\}+\mathrm{E}^{2}\{X\}
$$

and under the prerequisite that $n_{\Re}(t)$ and $n_{\Im}(t)$ have the same properties, it can be stated that

$$
\mathrm{E}\left\{X_{1}^{2}\right\}=\mathrm{E}\left\{X_{2}^{2}\right\}=\operatorname{Var}\left\{X_{1}\right\}=\operatorname{Var}\left\{X_{2}\right\}
$$

Therefore,

$N=2 r_{0}^{2} \operatorname{Var}\left\{X_{1}\right\}$

For further evaluation, the integrals are temporarily approximately replaced by sums. The reason is that in the continuous integral two "samples" of the noise process are directly adjacent to each other and therefore correlated. In reality, samples are taken with non-zero intervals between them. Assuming that those intervals are great enough, which is satisfied in many cases in reality, two samples of the noise process can be treated as uncorrelated. With the substitution

$f(x)=2 k r(\alpha(x))$,

the variance of $X_{1}$ can be evaluated as

$$
\begin{aligned}
& \operatorname{Var}\left\{X_{1}\right\} \\
& =\operatorname{Var}\left\{\int_{-x_{0}}^{x_{0}} \frac{n_{\Re}(t) \cos (f(x))-n_{\Im}(t) \sin (f(x))}{D(\alpha(x))} d x\right\} \\
& \approx \operatorname{Var}\left\{\sum_{l=-l_{0}}^{l_{0}} \frac{n_{\Re}(t) \cos (f(l \Delta x))}{D(\alpha(l \Delta x))} \Delta x\right. \\
& \left.\quad-\sum_{l=-l_{0}}^{l_{0}} \frac{n_{\Im}(t) \sin (f(l \Delta x))}{D(\alpha(l \Delta x))} \Delta x\right\} \\
& =\Delta x^{2} \operatorname{Var}\left\{\sum_{l=-l_{0}}^{l_{0}} \frac{n_{\Re}(t) \cos (f(l \Delta x))}{D(\alpha(l \Delta x))}\right\} \\
& +\Delta x^{2} \operatorname{Var}\left\{\sum_{l=-l_{0}}^{l_{0}} \frac{-n_{\Im}(t) \cos (f(l \Delta x))}{D(\alpha(l \Delta x))}\right\}
\end{aligned}
$$

$$
\begin{aligned}
& =\Delta x^{2} \sum_{l=-l_{0}}^{l_{0}} \operatorname{Var}\left\{\frac{n_{\Re}(t) \cos (f(l \Delta x))}{D(\alpha(l \Delta x))}\right\} \\
& \quad+\Delta x^{2} \sum_{l=-l_{0}}^{l_{0}} \operatorname{Var}\left\{\frac{-n_{\Im}(t) \cos (f(l \Delta x))}{D(\alpha(l \Delta x))}\right\} \\
& =\Delta x^{2} \operatorname{Var}\left\{n_{\Re}(t)\right\} \sum_{l=-l_{0}}^{l_{0}}\left(\frac{\cos (f(l \Delta x))}{D(\alpha(l \Delta x))}\right)^{2} \\
& \quad+\Delta x^{2} \operatorname{Var}\left\{n_{\Im}(t)\right\} \sum_{l=-l_{0}}^{l_{0}}\left(-\frac{\sin (f(l \Delta x))}{D(\alpha(l \Delta x))}\right)^{2} \\
& =\frac{1}{2} \cdot \Delta x \cdot P_{\mathrm{n}}^{\prime} \cdot \sum_{l=-l_{0}}^{l_{0}} \frac{1}{D^{2}(\alpha(l \Delta x))} \Delta x \\
& \approx \frac{1}{2} \cdot \Delta x \cdot P_{\mathrm{n}}^{\prime} \cdot \int_{-x_{0}}^{x_{0}} \frac{1}{D^{2}(\alpha(x))} d x .
\end{aligned}
$$

With the substitution

$x=r_{0} \tan \alpha, \quad d x=\frac{r_{0}}{\cos ^{2}(\alpha)} d \alpha$

and Eqs. (8), (6), (7), (A16), the the maximum achievable signal-to-noise ratio can be written as in Eq. (14).

\section{Appendix B Optimization}

Content of this section is the minimization of expression (9) with the boundary condition (12) by means of Lagrange multipliers and the calculus of variations. With the substitution

$y(\alpha)=D(\alpha) \cos (\alpha)$,

the Lagrange functional

$$
\begin{aligned}
F(\alpha) & =\int_{-\alpha_{0}}^{\alpha_{0}}\left(\frac{1}{y^{2}(\alpha)}+\lambda y(\alpha)\right) d \alpha-\lambda \frac{2 \pi}{\beta_{0}} \\
& =\int_{-\alpha_{0}}^{\alpha_{0}} f(y(\alpha)) d \alpha-\lambda \frac{2 \pi}{\beta_{0}}
\end{aligned}
$$

with

$f(y(\alpha))=\frac{1}{y^{2}(\alpha)}+\lambda y(\alpha)$

can be formed. A necessary condition for $D$ being an extremal is

$f_{y}=0$.

This condition is fulfilled by

$D(\alpha)=\left(\frac{2}{\lambda}\right)^{\frac{1}{3}} \cdot \frac{1}{\cos (\alpha)}$. 
F. Gerbl and E. M. Biebl: On the optimum antenna pattern for widebeam radar reflectivity estimation

Using the boundary condition (12), $\lambda$ can be determined to be

$\lambda=2\left(\frac{\beta_{0} \alpha_{0}}{\pi}\right)^{3}$.

With Eq. (B5), the optimum pattern as given in Eq. (13) follows.

Acknowledgement. The authors would like to thank K. Frick, Institute of Computer Science, University of Innsbruck, Austria, for the fruitful correspondence regarding the optimization method.

\section{References}

Ansorge, R. and Oberle, H. J.: Mathematik für Ingenieure, Band 2, Wiley, Berlin, 2000.

Gerbl, F., Biebl, E. M.: SNR Considerations for Widebeam, ShortRange Synthetic Aperture Radar Processing, GeMiC, 2006.

Orfanidis, S. J.: www.ece.rutgers.edu/ $\sim$ orfanidi/ewa/ch14.pdf, 2004. 